\title{
À la recherche du conservatoire industriel genevois (1834-1888)
}

In search of the conservatoire industriel of Geneva (1834-1888)

\section{Sylvain Wenger}

\section{(2) OpenEdition}

1 Journals

Édition électronique

URL : http://journals.openedition.org/artefact/888

DOI : $10.4000 /$ artefact.888

ISSN : 2606-9245

Éditeur :

Association Artefact. Techniques histoire et sciences humaines, Presses universitaires du Midi

Édition imprimée

Pagination : 177-188

ISBN : 978-2-7535-7305-5

ISSN : 2273-0753

\section{Référence électronique}

Sylvain Wenger, « À la recherche du conservatoire industriel genevois (1834-1888) », Artefact [En ligne], 6 | 2017, mis en ligne le 31 mai 2018, consulté le 07 mars 2020. URL : http://journals.openedition.org/ artefact/888; DOI : https://doi.org/10.4000/artefact.888 


\section{À la recherche du conservatoire industriel genevois (1834-1888)}

Sylvain WENGER*

\section{Résumé}

Des diverses institutions à vocation éducative établies par la Société des arts de Genève (1776), le conservatoire industriel, actif entre 1836 et 1888, demeure encore presque méconnu. Cette contribution propose des éclairages sur les principaux jalons historiques de ce dispositif et suggère que l'enrichissement de sa collection répond au besoin éprouvé par ses responsables de faciliter l'acquisition et la mise à disposition de savoirs industriels développés à l'échelle locale et à l'étranger.

Mots-clés : conservatoire, formation technique, objets, savoir, Société des arts (Genève).

\section{Abstract. In search of the conservatoire industriel of Geneva (1834-1888)}

Among the multiple institutions with an educational aim set up by the Society of Arts of Geneva (1776), the conservatoire industriel, which operated between 1836 and 1888, remains the least well known. The article highlights the institution's major historical milestones and suggests that the growth of its collections was largely driven by what the directors of the institution felt was a need to facilitate the acquisition and spread of industrial knowledge developed locally and abroad.

Keywords : conservatory, knowledge, objects, technical training.

\footnotetext{
*. Docteur ès sciences économiques et sociales mention histoire économique, Sylvain Wenger a soutenu, en 2016, à l'université de Genève, sa thèse intitulée Industrialisation, innovation et institutions du savoir : une perspective genevoise (1750-1850). Il est membre partenaire du Centre Alexandre Koyré - EHESS (Paris), membre externe de l'Institut d'histoire économique Paul Bairoch - Université de Genève et dirige actuellement un projet de valorisation des ressources historiques de la Société des Arts de Genève. Contact : [sylvainwenger.unige@gmail.com].
} 
Dans sa séance du lundi 21 mai 1888, le Comité de la Classe d'industrie et de commerce de la Société pour l'avancement des arts de Genève entérine la cession des plus de trois mille objets constituant son conservatoire industriel. La collection se voit alors scindée en deux parties, pour être remises à deux établissements de formation technique dépendant des instances publiques, à savoir l'École professionnelle et l'École $\mathrm{d}^{\prime}$ horlogerie ${ }^{1}$. Tout en mettant un terme à plus de cinquante ans d'activité du conservatoire, cette décision entend garantir la pérennité des objets dont il se compose.

Cette contribution présente un état des lieux préliminaire de la recherche sur le conservatoire industriel genevois. Elle éclaire les principaux jalons du développement de l'établissement et suggère, en filigrane, que les choix d'enrichissement de ses collections répondaient largement au besoin éprouvé par ses responsables successifs de faciliter l'acquisition et la mise à disposition de savoirs industriels développés à l'échelle locale et à l'étranger.

$\mathrm{La}$ trajectoire $\mathrm{du}$ conservatoire industriel genevois prend place dans le contexte des activités de la Société pour l'avancement des arts, des manufactures et de l'agriculture de Genève ${ }^{2}$, créée en 1776 dans l'intention de soutenir le développement industriel local en favorisant " l'émulation des Artistes » et la diffusion des "lumières qui peuvent leur être utiles ${ }^{3} »$. Cette institution s'inscrit dans le cadre du mouvement associatif européen qui voit la multiplication d'innombrables sociétés "économiques", « utilitaires » ou "d'émulation » à partir du premier tiers du $\mathrm{XVIII}^{\mathrm{e}}$ siècle ${ }^{4}$, au sein desquelles, parmi d'autres activités, on examine les dernières propositions techniques locales et étrangères, on ouvre des bibliothèques spécialisées, on offre des récompenses, on organise des expositions de produits et on se préoccupe d'améliorer la formation des fabricants.

$C^{\prime}$ est dans la perspective du soutien à la formation technique que des " musées » ou " conservatoires » industriels sont créés à partir de la fin du xvIII siècle, à l'image du Conservatoire national des arts et métiers, ouvert à Paris en 1794, ou à celle de son homologue mis en place à Bruxelles à la fin des années $1830^{5}$. De tels dispositifs, tantôt publics, privés ou mixtes, se multiplient davantage encore dans la seconde moitié du xIX ${ }^{\mathrm{e}}$ siècle. À la suite du South Kensington Museum - ce musée public d'art et d'industrie ouvert à Londres en 1857 dans la continuité de l'exposition internationale de $1851^{6}$-, diverses villes européennes dont Vienne, Hambourg, Stockholm, Budapest, Berlin et Paris suivent le mouvement. La Suisse n'est pas étrangère à ce phénomène puisqu'entre 1862 et 1889 , des musées industriels, d'art industriel ou d'arts décoratifs sont créés à Lausanne, Berne, Winterthur, Zürich, Saint-Gall, La Chaux-de-Fonds et Fribourg ${ }^{7}$. Comme nous allons le voir, le cas genevois présente une temporalité singulière. 


\section{Origines et intentions du projet}

À Genève, c'est au sein de la Classe d'industrie et de commerce de la Société des arts locale que le projet d'un conservatoire prend forme ${ }^{8}$. En février 1834, une commission créée pour examiner la proposition de l'un de ses membres, un certain Colladon, sur " la formation d'un musée industriel ", se prononce favorablement. En accord avec la municipalité, on profitera de l'opportunité qui se présente alors de disposer $\mathrm{d}^{\prime}$ « un local propre à réunir une [telle] collection ${ }^{9}$ ». À l'issue de la séance, il est décidé de créer l'établissement et de donner mandat à ladite commission de déterminer « la nature de la collection qui sera à former ${ }^{10} »$.

À l'origine du projet, Jean-Daniel Colladon (1802-1893), un des plus importants ingénieurs genevois de son siècle $^{11}$. De qui s'agit-il? Diplôme de droit en poche, il quitte Genève en 1824 pour aller étudier les mathématiques et la physique à Paris, où il est assistant $\mathrm{d}$ 'André Ampère et voit ses travaux sur la compressibilité des liquides récompensés par le grand prix de l'Académie des sciences (1827). Colladon participe à la création de l'École centrale des arts et manufactures (1829) où il enseigne jusqu'en 1839. Il rentre ensuite en Suisse et fait carrière dans le génie civil. Enseignant à l'Académie de Genève, il joue un rôle central dans le développement des perforatrices à air comprimé employées dans le percement des tunnels - le Gothard, notamment, dans les années 1870 -, ce qui lui vaudra le prix Fourneyron de l'Académie des sciences de Paris en $1884^{12}$. C'est en somme un acteur particulièrement bien connecté aux réseaux techniques et scientifiques parisiens qui importe à Genève l'idée de créer un conservatoire industriel.

La mission assignée au conservatoire genevois consiste à soutenir l'« enseignement industriel ${ }^{13} »$. La création de l'établissement parachève en réalité un processus d'institutionnalisation de la formation technique genevoise amorcé par la Société des arts au début des années $1820^{14}$, avec notamment la création des écoles d'horlogerie, dès 1824, et de l'École industrielle et commerciale, en $1830^{15}$. Le rôle de l'institution est soustendu par un discours pédagogique selon lequel l'appréhension physique et visuelle de techniques industrielles anciennes, et nouvelles, est décisive pour l'amélioration des compétences des apprentis ainsi que des fabricants établis. Dans cette perspective, le président de la Classe d'industrie et de commerce, le physicien Auguste de La Rive (1801-1873), en exercice en 1840, réitère l'intention d'en faire «à la fois un musée historique [des] industries passées, et une école de perfectionnement par une collection de bons modèles, [des] industries présentes et peut-être [des] industries futures ${ }^{16}$.»

Lors des séances de travail préparatoires, où l'on discute de la nature de la collection en devenir, il est d'abord proposé de se concentrer sur « les appareils d'expériences et de démonstrations, nécessaires pour les cours de Mécanique, de Physique et de Chimie de l'École Industrielle »; les opinions sont cependant globalement défavorables, sachant que le musée académique local dispose déjà de tels appareils, que la Classe d'industrie pourrait continuer à emprunter. 
Au fil des discussions, on tendra plutôt à privilégier la réunion de "modèles de machines et appareils, appliqués et utiles à l'industrie ». De plus, il est question de rapatrier des objets appartenant à la Société mais déposés au Cabinet de mécanique du musée académique, et d'acquérir des pièces auprès du gouvernement et de particuliers ${ }^{17}$. Quoi qu'il en soit, le premier projet de règlement $\mathrm{du}$ conservatoire, daté de juin 1834, stipule qu'il s'agit de " réunir des modèles de machines, appareils ou instrumens [sic] propres à donner une idée juste de l'état actuel des arts mécaniques et industriels", et de "former une collection spéciale des pièces et modèles propres à montrer la série des inventions relatives à la mesure du temps, dès l'origine de $l^{\prime}$ art jusqu'à nos jours ${ }^{18}{ }^{\text {». }}$

\section{De la maison municipale à l'ancien grenier à blé}

Le conservatoire ouvre ses portes pour la première fois en juillet 1836 dans une salle mise à disposition par la municipalité19, rue de la Corraterie, à proximité du musée Rath, le siège de la Société des arts de l'époque ${ }^{20}$. Parmi les premiers objets à intégrer la collection, entre 1835 et 1840, figurent un " chronomètre fait à Liverpool », des " modèles de roues hydrauliques perfectionnées ", une " très-petite machine locomotive fonctionnant par le moyen de la vapeur, construite sur le modèle de celles qui remorquent les voitures sur le chemin de Paris à Saint-Germain », un modèle d' « engrenage hélicoïdal », une "pendule astronomique d'ancienne construction", ou encore une "machine à éprouver les fers ». À partir de 1839, la Classe d'industrie dispose d'une seconde salle prêtée par la municipalité, qu'elle emploie pour entreposer " des dessins de machines, des modèles en nature, ou des produits de l'industrie genevoise ». Le statut précis de cette annexe du conservatoire reste cependant à éclaircir ${ }^{21}$.

En 1842, la maison municipale de la Corraterie est vendue ${ }^{22}$ et le conserva- toire est déplacé dans un ancien grenier à blé municipal, situé dans le quartier de Rive $^{23}$. Au cours de cette décennie, les collections continuent d'être enrichies au moyen de dons et d'acquisitions, comme un "modèle de danaïde, soit roue hydraulique » (1842), " de grands dessins descriptifs de machines, peints à $l^{\prime}$ huile, qui se vendent à Paris à un prix assez modique » (1843), des " modèles de locomotives, de rails, de sleepers et autres objets relatifs à la construction et à l'usage des chemins de fer » (1844), un " outil pour couper les feuilles de mûrier » (1845), un « modèle d'outil destiné à tailler les pignons hélicoïdaux [et] les pignons droits » (1846), des "modèles de ponts » (1847), « deux anciennes lampes de notre célèbre compatriote Ami Argand, inventeur des lampes à courant d'air » (1848) ${ }^{24}$. Il arrive que la Classe d'industrie fasse expressément fabriquer des pièces à des artisans locaux, par exemple des modèles agrandis de « deux échappements en métal, l'un duplex, l'autre à ancre ", commandés, en 1843 , à un certain Séchehaye ${ }^{25}$. 
S'il est vrai qu'au moment de la création du conservatoire, la Société des arts et les autorités locales collaborent volontiers, en revanche des dissensions apparaissent une dizaine d'années plus tard dans le contexte de la révolution radicale de 1846. Le nouveau régime, emmené par son leader James Fazy (1794-1878), considère que l'association est trop élitiste et lui reproche de perpétuer les prérogatives de l'oligarchie genevoise. En 1850, un arrêté ordonne sa dissolution et commande au Conseil administratif de la ville la récupération du musée Rath et la confiscation d'une partie de ses biens ${ }^{26}$. Le 20 mai 1851, la Société est effectivement expulsée du musée Rath, non sans avoir opposé de la résistance. Il est saisissant de constater qu'en dépit des remous qui bouleversent l'institution mère, le conservatoire n'en poursuit pas moins son activité. Il continue en effet « à être ouvert une fois par semaine "; à cette période, apprend-on dans un rapport annuel, la Classe d'industrie " n'a pas été troublée dans la paisible jouissance de cette précieuse collection » et « a pu continuer à en faire jouir le public ${ }^{27} »$. On ne cesse, de plus, d'acquérir de nouveaux objets, tels qu'un " très-beau compas à engrenage, exposé dans le palais de cristal [...] du Locle » et une " série de pièces pour démonter la construction des échappements à ancre [...], tels qu'on les construit en Angleterre $»(1851)^{28}$.

En 1852, la Société retrouve son statut légal de fondation, ainsi qu'une partie des collections qui lui avaient été confisquées en mai $1851^{29}$. Si aucune acquisition n'est réalisée cette annéelà, en revanche la commission chargée $\mathrm{du}$ conservatoire industriel s'attelle à la réalisation d'un inventaire complet visant à faire « garantir [la collection] contre l'incendie ${ }^{30} »$. Dès l'exercice suivant, le conservatoire recommence à être enrichi. On acquiert ainsi, par exemple, des « dessins de ponts » et un «modèle de charpente en fer » en 1853; une « toiture en fer zinqué » et un " grand modèle de règle à calcul » (1854); un « appareil pour la vinification ", un «bluttoir », un " moulin à bras » et un "pétrin mécanique ", offerts par la Classe d'agriculture, ainsi qu'une " turbine » $(1855)^{31}$; une " glace argentée » et de nouveaux modèles de "ponts en charpente » et un " modèle de clocher » (1857); un " modèle du Polytrope de Magnus » fabriqué à Aarau et un " planimètre polaire » de Schaffhouse (1858); des " modèles d'hélice " et une " collection de dessins de grande dimension relatifs à la technologie et à la physique » (1859); une " ancienne pendule à godets ", et un " poële en fonte » utilisé « aux ateliers d'Yverdon ", afin de "brûler les déchets de bois », et enfin " 33 tableaux technologiques de très-grand format, publiés par le professeur Knapp, à Munich ", ainsi que "deux tableaux de machines marines du professeur Ortolan, de Brest », lors de l'exercice $1860^{32}$. 


\section{Le conservatoire au palais de l'Athénée}

Si la Société maintient une partie de ses activités au cours des années 1850, ce n'est qu'au milieu de la décennie suivante qu'elle retrouve une stabilité durable. En 1864, la Société des arts s'installe dans le palais de l'Athénée, construit et mis à sa disposition par le financier philhellène d'origine lyonnaise Jean-Gabriel Eynard (1775-1863) et son épouse, Anna EynardLullin (1793-1868). Le conservatoire y est installé dans un local spacieux, comme le décrit le président de l'époque, le botaniste Alphonse de Candolle (18061893), dans le discours prononcé à l'occasion de l'inauguration du palais :

« Les salons de l'aile gauche, au rez-de-chaussée, serviront à la bibliothèque de la Classe d'industrie et aux séances ordinaires des Classes. Les bibliothèques d'agriculture et des beaux-arts sont dans une chambre très-commode de l'entresol. Une salle au-dessous de celle-ci et non moins vaste [...] est destinée au conservatoire d'instruments de la Classe d'industrie ${ }^{33}$.»

À l'Athénée ${ }^{34}$, le conservatoire reprend $\mathrm{du}$ service tout en faisant l'objet d'une importante réorganisation, privilégiant la dimension muséale et tendant à favoriser l'élargissement des publics cibles. $\mathrm{Au}$ cours de l'exercice 1866, la Classe d'industrie décide ainsi « de joindre à son Conservatoire de machines un petit musée technologique, où les divers produits de l'industrie seront exposés suivant $l^{\prime}$ ordre de leurs phases successives ${ }^{35}{ }$. Les responsables se féliciteront bientôt que des visiteurs différents du « public habi- tuel ", constitué d'apprentis, d'ouvriers et d'étrangers de passage, consultent de plus en plus souvent les collections. À ce stade de nos investigations, il demeure toutefois délicat d'évaluer précisément la nature de ces changements ${ }^{36}$.

Durant cette période, le discours soutenant le développement de l'établissement fait de plus en plus fréquemment référence à des institutions étrangères, comme autant de sources d'inspiration. On le constate, par exemple, dans un rapport relatif à l'exercice 1866 révélant que les responsables du conservatoire ont souhaité «s'associer [...] à la grande idée anglaise de l'instruction par les yeux, qui provoque une curiosité de bon aloi et le goût de lectures sérieuses. Ce que Londres a créé dans son grand établissement de Kensington, ce que $\mathrm{M}^{\mathrm{me}}$ de Rumine a imité à Lausanne, nous avons voulu le reproduire dans notre ville ${ }^{37} »$; ou, dans celui de 1868, lorsque la Classe d'industrie " introduit à l'Athénée, au grand profit de la population ouvrière, un enseignement par les affiches, à l'instar de ce que les Anglais ont fait au musée de South Kensington et de ce qu'on a imité à Lausanne, à Annecy, comme à Vienne et à Berlin ». Aussi, du point de vue des types d'objets conservés, l'intérêt se fait progressivement plus prononcé pour les matériaux, comme en témoigne, lors du même exercice, l'aménagement de vitrines abritant des échantillons des "principales matières premières utilisées dans les diverses industries ", auxquels on adjoint des informations sur « la suite des transformations qu' on leur fait subir pour les métamorphoser en produits utilisables ». 
Au début des années 1870, la réorganisation du conservatoire est achevée. C'est pour cette période qu'il nous est pour la première fois donné de disposer de renseignements sur le nombre d'objets formant les collections. L'établissement, que l'on qualifie dès lors plus volontiers de " musée » que de " conservatoire ", compte alors « 3621 objets ou séries d'objets, répartis en onze classes suivant les branches de l'industrie auxquelles ils se rapportent ${ }^{38}$ ». S'il est bel et bien question d'objets, le conservatoire abrite également des substances, comme les responsables l'expriment en 1875 , en soulignant que « ce musée fort instructif [...] a pour but de faire connaître l'origine, la nature et le mode de fabrication d'une foule d'objets ou de substances que nous employons tous les jours ${ }^{39} »$.

D'un côté, eu égard au rythme d'enrichissement des collections, il semble quel'activité du conservatoire soit encore relativement soutenue au début des années 1870. On note, par exemple, lors de l'exercice 1872, la réception d'un " modèle de perforatrice Sommeiller ", illustrant l'intérêt particulier du moment pour les techniques de percement des tunnels ferroviaires, ainsi que $\mathrm{d}^{\prime}$ « un mouchoir chinois très fin ", de "broderies sur cuir de Russie », $d^{\prime}$ " " une balance à peser les lettres », d'une « belle pépite houillère » ou de " photographies industrielles ». En 1873, des articles sont rapportés de l'exposition universelle de Vienne et, en 1878, le conservatoire reçoit « une bouteille de verre [...] transformée en sorte de grès dur par l'effet d'un violent incendie » de prison à Paris, en 1871 , et « un modèle de réservoir à pétrole à l'abri de l'incendie ${ }^{40}$ ».
D'un autre côté, dans le même temps, des signes indiquent que l'intérêt pour le dispositif tend peu à peu à décliner. D'abord, dès l'exercice 1870, les responsables de l'établissement font le constat lucide de la prise en charge progressive mais inéluctable de tous les aspects de l'enseignement industriel par l'État, comme en témoigne l'extrait de rapport annuel suivant :

" Les leçons publiques, qui ont lieu tous les soirs à l'Hôtel-de-Ville, font une concurrence décisive aux enseignements techniques que notre Association avait l'habitude d'offrir aux industriels. Nous constatons avec regret l'absence d'un nombre suffisant d'auditeurs aux cours donnés cet hiver sur l'hydraulique, l'art des constructions et le droit commercial, malgré le zèle et le talent des professeurs. Nous pensons qu'il ne faut plus persévérer dans une voie que nous avons sans doute eu l'honneur d'inaugurer, mais dont l'État a pris désormais la direction et la responsabilité ${ }^{41}$. »

De surcroît, le développement de l'offre de formation professionnelle publique mené à cette période s'accompagne, dès le milieu des années 1870 au moins, d'un projet de «musée industriel ». Ainsi lit-on dans le compte rendu municipal de l'année 1875 que le mauvais état des locaux de l'École d'horlogerie fait espérer à la commission municipale ad hoc la mise à disposition d'un nouveau bâtiment incluant « tous les espaces nécessaires pour les salles de cours, les ateliers des élèves et l'installation d'un musée spécial industriel ${ }^{42}$ ». De 
fait, dès 1881, à la suite de la réorganisation de cet établissement de formation, des pièces horlogères sont progressivement réunies dans les nouveaux locaux, rue Necker, afin de former la base d'un " musée » appelé à « compléter l'organisation de l'Académie professionnelle ${ }^{43}$ ».

Un autre motif de préoccupation pour les responsables du conservatoire réside dans le manque d'espace disponible. Dès 1874, ils appellent de leurs vœux " un local plus vaste et plus commode » qu'ils espèrent obtenir dans le cadre du « centenaire de la Société ", prévu en 1876, sans quoi, "faute de place", il ne sera bientôt plus possible d'augmenter les collections $^{44}$.

Cela étant, jusqu'au milieu des années 1880 au moins, rien n'annonce concrètement la fermeture prochaine $\mathrm{du}$ conservatoire. Les collections continuent d'accueillir quelques nouvelles pièces, comme, en 1882, " 20 échantillons sur différentes qualités de laines et graines d'Australie ", ou, en 1886, des "vis de revolver ", une "paire de mocassins brodés par des femmes indiennes de Manitoba (Amérique du Nord) », « deux moteurs électriques à lampes ", " une collection de sonneries électriques, des piles et autres objets ${ }^{45} »$. De fait, le directeur du conservatoire, le professeur de physique Élie-François Wartmann (18171886), en poste depuis 1871 , réitèrera sa foi en l'institution en 1881 comme lieu fort de l'activité inventive, comme on le constate dans une note où il insiste sur l'importance de poursuivre l'acquisition de pièces d'horlogerie afin d'attirer "l'attention des ouvriers qui visitent le Conservatoire, et [d'éveiller] chez quelques-uns le désir d'améliorer les procédés, en les poussant peut-être dans la voie de l'invention. Sortir de la routine, écrit-il, c'est faciliter le progrès ${ }^{46}{ }^{\prime}$.

La situation connait un tournant important dans les années 1886 et 1887, à la suite du décès de Wartmann, principal moteur de la réorganisation de l'établissement lors de son installation à $1^{\prime}$ Athénée ${ }^{47}$. Le rapport annuel de l'exercice 1886 s'ouvre par une formule teintée de sarcasme témoignant de la faible activité du conservatoire : "S'il est vrai que les gens les plus heureux sont ceux dont l'histoire est monotone, le conservatoire industriel doit jouir sur cette terre d'une béatitude parfaite ${ }^{48}$. » On y évoque également la question $\mathrm{du}$ " déplacement $\mathrm{du}$ conservatoire ", en discussion depuis plusieurs années avec les autres sections de la Société des arts - les Classes d'agriculture et des beaux-arts - qui souhaitent disposer de la place dévolue jusque-là au conservatoire.

On connaît encore mal les circonstances précises de la fermeture de l'établissement, au cours de l'année 1888. Tout au plus sait-on que les collections sont remises à l'École professionnelle et à l'École d'horlogerie, des institutions gérées respectivement par la Ville et par l'État de Genève. Des renseignements sur la répartition des objets nous sont connus par un projet de convention daté du mois de mai 1888, liant la Société des arts aux autorités locales ${ }^{49}$. Ce document, qui s'accompagne d'un inventaire manuscrit détaillé des objets destinés à l'École d'horlogerie, constitue la seule source connue à ce jour comportant des informations circonstanciées sur l'organisation générale de la collection. On y lit que cette dernière est alors catégorisée selon les onze « classes » suivantes : mécanique, métaux, chimie, agriculture, 
alimentation, bâtiment, mobilier, vêtement, hygiène et médecine, décoration et enseignement. Ces derniers éléments sont précieux pour décoder la seule image du conservatoire dont on dispose à ce jour (fig. 20, cahier couleur). Le

\section{Perspectives de recherche}

Notre compréhension de la trajectoire $\mathrm{du}$ conservatoire industriel genevois et de ses dynamiques internes demeure encore fragmentaire. L'état de nos connaissances pourrait, cela dit, évoluer dans un avenir proche, étant donné que, depuis 2016, la Société des arts de Genève a initié un projet de conservation et de valorisation de ses archives susceptible de faire émerger du matériel resté jusqu'ici dormant. De plus, du matériel conservé dans les archives de la ville de Genève mérite encore examen, qui permettrait de saisir plus avant les processus menant à la reprise du dispositif par les instances publiques.

Dans le cadre d'investigations ultérieures, il serait judicieux d'examiner l'évolution des publics de l'institution, de même que ses modes de financement; de procéder à un recensement systématique des objets qui viennent régulièrement enrichir le conservatoire afin, par exemple, de mettre au jour l'évolution des stratégies d'enrichissement des collections et d'évaluer la vitesse à laquelle les informations sur les derniers progrès industriels réalisés à l'étranger parvenaient aux acteurs genevois; et, si les sources encore en cours d'inventaire le permettent, d'examiner les modes de classification des objets, afin de ques- cliché de cette probable mise en scène fut réalisé autour de 1880 afin d'être envoyé à l'exposition universelle de Melbourne (1880-1881) dans l'intention d'y représenter l'industrie genevoise ${ }^{50}$. L'enquête continue.

tionner la manière dont les contemporains se représentaient l'organisation des différents champs de la connaissance industrielle. Ces questions pourraient être avantageusement mises en comparaison avec d'autres expériences qui nous sont déjà connues ${ }^{51}$.

Pour l'heure, cette contribution met en évidence que les responsables du conservatoire industriel genevois se préoccupaient de favoriser l'acheminement de connaissances locales et étrangères pouvant être utilisées à des fins industrielles et d'en faire connaître l'existence aux apprentis, aux fabricants et, plus largement, à toutes les personnes intéressées aux techniques à Genève. Dans cette perspective, ce dispositif peut être considéré comme un important mécanisme de soutien au développement industriel local. 


\section{Notes}

1. Je remercie Noam Cochin, Ella Meister et Annina Pinösch pour leur contribution à la réalisation de cet article.

2. La Société des arts de Genève est créée par un cercle de savants et d'artisans, réuni depuis 1770 environ, à l'initiative du physicien naturaliste Horace-Bénédict de Saussure (1740-1799).

3. Archives de la Société des arts (ensuite ASdA), Prospectus de l'établissement de la Société pour l'encouragement des Arts dans la ville et le territoire de la République de Genève, Genève, 1776.

4. Par exemple, la Royal Society for the encouragement of Arts, Manufactures \& Commerce de Londres (1754), l'Ökonomische Gesellschaft de Berne (1759), ou la Société libre d'émulation de l'Abbé Baudeau de Paris (1776). Sur le cas helvétique, voir Emil ERNE, Die Schweizerischen Sozietäten : Lexikalische Darstellung der Reformgesellschaften des 18. Jahrhunderts in der Schweiz, Zürich, Chronos, 1988.

5. Voir notamment Claudine FonTANoN, « Les origines du Conservatoire national des arts et métiers et son fonctionnement à l'époque révolutionnaire (1750-1815) ", Les cahiers d'histoire du Cnam, n 1, Paris, Cnam médias, 1992, et Claudine FontANON et André GRElon, Les professeurs du Conservatoire national des arts et métiers : dictionnaire biographique, 1794-1955, Paris, Institut national de recherche pédagogique, 1994, et, plus récemment, Lionel DufAux, L'Amphithéâtre, la galerie et le rail. Le Conservatoire des arts et métiers, ses collections et le chemin de fer : diffusion des connaissances techniques et de l'innovation au XIX siècle, thèse de doctorat non publiée, École des hautes études en sciences sociales (Centre A. Koyré), Paris, 2015. Sur le cas belge, on se référera à Marie-Christine CLAEs, «Marcellin Jobard et le musée royal de l'industrie de Bruxelles », Artefact, n 5, 2016, p. 59-75.

6. Il est rebaptisé Victoria and Albert Museum en 1889 ; Bruce Robertson, « The South Kensington Museum in context : an alternative history ", Museum and society, 2 (1), 2015, p. 1-14.

7. Ces institutions prennent des formes variables selon les régions et les périodes, comme le pointe Isaline Deléderray-Oguey qui souligne aussi la difficulté de clairement les distinguer; Isaline DelÉderray-OgueY, « Les musées industriels en Suisse et le Conservatoire national des arts et métiers de Paris, un modèle parmi d'autres ? ", Cahiers d'histoire du Cnam, n 5, 2016, p. 77-78.

8. Les archives de la Société des arts de Genève (ASdA) sont en cours d'inventaire dans le cadre d'un projet de conservation et de valorisation des collections de l'institution initié en 2016.
9. ASdA, Classe d'industrie, correspondance 1822-1847, rapport de la commission nommée par la Classe d'industrie, dans sa séance du janvier 1834, pour examiner la proposition faite par D. Colladon, concernant la formation d'un musée industriel, Genève, 19 février 1834. Commission composée du P ${ }^{r}$ G. Maurice, et Colladon, Dufour colonel, Marcet et Morin pharmacien.

10. L'intérêt pour le rassemblement d'objets industriels est, cela dit, antérieur à la constitution du conservatoire; on sait par exemple que la Société des arts reçoit, en 1800, des renseignements à ce sujet à la suite de la fondation de la Royal Institution of Great Britain, créée à Londres en 1799 : « Le Président [M.-A. Pictet] présente à la Société, de la part du [Comte] de Rumford, un Extrait du plan \& des Reglemens de l'Institution Royale qu'il a formée à Londres pour recevoir tous les modèles de machines utiles aux arts \& à l'économie domestique \& pour donner des cours de physique \& de chimie. ", ASdA, procès-verbaux des assemblées générales et des séances du comité, IV, 1799-1817, Genève, 18 août 1800, p. 54 .

11. Serge PAquier, " L'apport de Jean-Daniel Colladon à la convergence des sciences et des techniques au $\mathrm{XIX}^{\mathrm{e}}$ siècle ", in Laurence-Isaline Stahl Gretsch, Marc J. Ratcliff (éd.), Mémoires d'instruments, Genève, S. Hurter, 2011, p. 189-197.

12. Marino Buscaglia, «Jean-Daniel Colladon et les technologies de percement ", La recherche alpine et les transversales, Actes du congrès de l'Académie suisse des sciences naturelles, Publications de l'ASSN, nº 8, 2000, p. 133-137.

13. ASdA, rapport annuel du Comité d'industrie, fait par Macaire-Princep, remplaçant le $\mathrm{P}^{\mathrm{r}}$ De La Rive, président, Genève, 19 juin 1834. Les rapports du Comité d'industrie, largement exploités dans cette contribution, sont systématiquement encartés dans les procès-verbaux annuels de la Société des arts de Genève.

14. À propos de la priorité mise par la Société des arts sur la formation technique à la Restauration, Pierre Prevost, Mémoire sur les moyens d'améliorer l'éducation des jeunes gens destinés aux professions étrangères aux lettres : présenté au Conseil d'État par la Société pour l'avancement des arts, Genève; Paris, Paschoud, 1821. Et sur la prépondérance de la mission pédagogique des conservatoires, I. Deléderray-Oguey, « Les musées industriels en Suisse et le Conservatoire national des arts et métiers de Paris... », op. cit., p. 82-83.

15. Ces établissements créés par la Société des arts seront progressivement pris en charge par les autorités locales à partir de la seconde moitié des années 1830; Sylvain WENGER, Industrialisation, innovation et institutions du savoir : une perspec- 
tive genevoise (1750-1850), thèse de doctorat non publiée, université de Genève, 2016.

16. ASdA, rapport annuel du comité d'industrie, fait par le Pr De la Rive, Genève, 12 août 1841. On reconnaît là l'attrait de De la Rive pour ce que l'on appelle aujourd'hui la veille technologique, lui qui lancera quelques années plus tard un prix public destiné à récompenser " la découverte la plus utile à l'industrie genevoise » (AsdA, prix de six cents francs, fondé par De la Rive pour la découverte la plus utile à l'industrie genevoise, Genève, 1844).

17. ASdA, rapport..., op. cit., 19 février 1834.

18. Ibid.

19. Plus précisément à la nouvelle maison municipale de la Corraterie qui abrite aussi, dans ses premières années, des salles d'école, un hangar de matériel de lutte contre l'incendie, un local pour les poids et mesures, une salle pour la vente des objets saisis, des appartements d'officiers, une salle pour réunions militaires et une autre pour l'enseignement industriel; David RIPOLL, "La Maison municipale, rue de la Corraterie ", in Isabelle BRUNIER (dir.), Genève, espaces et édifices publics, Berne, Société d'histoire de l'art en Suisse, 2016 (Les monuments d'art et d'histoire du canton de Genève 4), p. 156.

20. Le musée Rath est placé sous la gestion de la Société des arts depuis sa construction en 1826; Maurice Pianzola et al., Le Musée Rath a 150 ans, Genève, Musée d'art et d'histoire, 1976; Danielle Buyssens, La question de l'art à Genève : du cosmopolitisme des Lumières au romantisme des nationalités, Genève, La Baconnière Arts, 2008, p. 336.

21. La salle est située à Rive; ASdA, rapports annuels du comité d'industrie (1835-1840), Genève.

22. Elle est réaffectée en édifice bancaire; D. RIPOLL, "La Maison municipale... », op. cit., 2016, p. 156.

23. ASdA, rapport annuel du Comité d'industrie, fait par le $\mathrm{Pr}^{\mathrm{r}}$ Gautier, président, Genève, 17 août 1843.

24. ASdA, rapports annuels du Comité d'industrie (1843-1849), Genève.

25. ASdA, rapport annuel du Comité d'industrie, fait par Samuel Viande, président, Genève, 8 août 1844 . Séance tenue dans la salle des séances du Grand Conseil. Il s'agit probablement de Charles Sechehaye (1817-1902).

26. Sur cet épisode, Jules Crosnier, La Société des Arts et ses collections, Genève, 1910, p. 68-71.

27. ASdA, rapport annuel du Comité d'industrie et de commerce, fait par J.-S. Viande, président, 11 août 1851. Séance tenue dans le local provisoire de la Société, rue des Chanoines.
28. ASdA, rapport annuel de la Classe d'industrie, fait par le $\mathrm{Pr}^{\mathrm{C}}$ Colladon, président, Genève, 30 juin 1852. Séance tenue dans le local de la Société, rue des Chanoines, $\mathrm{n}^{\circ} 123$.

29. Isabelle РАуот WunderLI, « L'art à Genève au dix-neuvième siècle ", in Karine Tissot (éd.), Artistes à Genève : de 1400 à nos jours, Genève, L'APAGE, 2010, p. xxviI.

30. Rapport annuel de la Classe d'industrie et de commerce, par Élie Ritter, président, Genève, 11 mai 1853. Cet inventaire ne nous est pas encore parvenu à ce jour.

31. En 1856, si aucune nouvelle acquisition n'est signalée, on s'enorgueillit en revanche de ce que " plusieurs modèles ont servi à l'instruction du jeune prince Alfred, second fils de la reine d'Angleterre, qui a passé six mois à Genève ».

32. ASdA, rapports annuels de la Classe d'industrie (1852-1861), Genève.

33. Alphonse de CANDolle, Discours de M. Alphonse de Candolle, président de la Société des arts, prononcé dans la séance générale du 5 janvier 1864 à l'occasion de l'installation de la Société dans l'Athénée, Genève, Société des arts, 1864, p. 24-25.

34. La Société en est propriétaire et y a toujours son siège de nos jours.

35. ASdA, rapport annuel de la Classe d'industrie et de commerce, par le $\mathrm{Pr}^{\mathrm{r}}$ Élie Wartmann, président, Genève, 23 mai 1867. Séance tenue à l'Athénée.

36. Et que penser, plus spécialement, du constat figurant dans le rapport de l'exercice 1868, selon lequel « les mères, accompagnées de leurs jeunes enfants, y prennent un intérêt extrême : il en est qui nous sont revenus à plusieurs reprises "? ASdA, rapport annuel de la Classe d'industrie et de commerce, par le Pr Élie Wartmann, président, Genève, 29 mai 1869.

37. ASdA, rapport annuel de la Classe d'industrie et de commerce, par le $\mathrm{Pr}^{\mathrm{E}}$ Élie Wartmann, président, Genève, 23 mai 1867.

38. ASdA, rapport annuel de la Classe d'industrie et de commerce, par Louis Soret, président, Genève, 23 mai 1872.

39. ASdA, rapport annuel de la Classe d'industrie et de commerce, par Gustave Rochette, viceprésident, Genève, 20 mai 1875.

40. ASdA, rapport annuel de la Classe d'industrie et de commerce, par J. Weibel, président, Genève, 25 mai 1878 .

41. ASdA, rapport annuel de la Classe d'industrie et de commerce, par Louis Soret, président, Genève, 23 mai 1872.

42. Archives de la ville de Genève (ensuite AVdG), Rapport de la Commission de l'École d'horlogerie, in Compte rendu de l'administra- 
tion municipale de la ville de Genève pour l'année 1875, p. 21.

43. AVdG, Comptes rendus de l'administration municipale de la ville de Genève pour les années 1881 à 1883. À propos de l'histoire de l'établissement, Eugène JAQuET, L'École d'horlogerie de Genève : 1824-1924, Genève, Atar, 1924, et Estelle FALlET, «École d'horlogerie Genève », in Estelle FAllet (éd.), Dix écoles d'horlogerie suisses : chefsd'euvre de savoir-faire, Neuchâtel, Éd. Simonin, 2010, p. 11-83.

44. ASdA, rapport annuel de la Classe d'industrie et de commerce, par Gustave Rochette, viceprésident, Genève, 20 mai 1875.

45. ASdA, rapport annuel de la Classe d'industrie et de commerce, par Adolphe Gautier, président, Genève, 31 mai 1883; ASdA, rapport annuel de la Classe d'industrie et de commerce, par H. Veyrassat, président, Genève, 2 juin 1887.

46. Je n'ai pas pu établir clairement le statut de cette note, mais il semble s'agir d'un message interne, ASdA, note de É. Wartmann datée du 15 septembre 1881, boîte «Conservatoire industriel $»$.

47. À propos de ses intérêts pour le développement technique, Élie F. WARTMANN, Notice historique sur les inventions et les perfectionnements faits à Genève dans le champ de l'industrie et dans celui de la médecine, Genève, H. Georg, 1873.

48. Ce rapport stipule pourtant que le nombre de visites enregistrées en 1886 est supérieur à celui de l'année précédente, mais il tempère aussitôt cette augmentation en signalant que le nombre de jours d'ouverture a augmenté, ASdA, rapport sur le Conservatoire industriel pour l'année 1886, Genève, 8 mai 1887.

49. Le document prévoit alors prudemment un dépôt « pour dix ans », ASdA, projet de convention relatif à la cession éventuelle du Conservatoire industriel, Genève, mai 1888; annexés, deux exemplaires d'un inventaire manuscrit des objets destinés à l'École d'horlogerie.

50. J'adresse mes remerciements à J.-D. Clerc, collectionneur et marchand d'affiches à Genève, pour m'avoir aidé à retrouver cette image en activant ses réseaux. Ma reconnaissance va aussi à Stéphane Fischer, conservateur adjoint au musée d'histoire des sciences de Genève, qui m'a signalé que plusieurs pièces figurant sur cette image sont aujourd'hui encore conservées dans les soussols de l'École de mécatronique industrielle de Genève.

51. Par exemple, sous l'angle de l'enrichissement des collections, à la lumière de MarieSophie CORCY, « La politique d'enrichissement des galeries du Conservatoire des arts et métiers (1849-1880) », Artefact, n 3, 2015, p. 165-182, ou de Chloé Sauvalle, Histoire d'une collection : de la Société d'encouragement pour l'industrie nationale au Conservatoire national des arts et métiers, mémoire de master 2 sous la dir. de M.-S. CorCy, P. CROzET et L. Hilaire-Pérez, université Paris DiderotParis 7, 2006; ou sous l'angle des publics, au regard de Samuel HAYAT, "Les savoirs et leurs publics : l'exemple du conservatoire des arts et métiers (19e-21 e siècles) ", Innovations, $n^{\circ} 52,2017$, p. 139-160. 